Among the first applications of timeresolved X-ray diffraction to a chemical reaction was the study of the structural changes that are associated with the photodissociation of the CO group in myoglobin, traced at the nanosecond timescale. Plech et al. have extended the diffraction technique to reach the picosecond timescale and track the photodissociation of iodine molecules dissolved in carbon tetrachloride. This reaction has been examined extensively as a model of the competition between two modes of recombination for the iodine atoms: whether the photodissociated atoms are trapped by the solvent and recombine with each other (called geminate recombination); or whether the iodine atoms escape their cage of solvent and recombine with other iodine atoms (non-geminate recombination).

Plech et al. used 150-fs laser pulses to excite the iodine solute, generating a mixture of electronic states that mainly dissociate to ground-state atoms. The excited iodine molecules that are formed have a bond length of $0.27 \mathrm{~nm}$, which stretches to $0.4 \mathrm{~nm}$ in less than a picosecond. Next, the molecule either dissociates into two iodine atoms or loses energy to the solvent, relaxing to an excited bound state on a timescale of 2.7 ns or to the ground state in about 180 ps. By sending intense 100 -ps pulses of X-rays into the iodine solution at varying time intervals after the laser excitation pulse, Plech et al. mapped changes in the bond lengths between iodine atoms and between neighbouring solvent molecules during the course of the reaction, through the series of diffraction patterns created.

On the question of geminate recombination, Plech et al. find that for only $14 \%$ of excited iodine molecules do the dissociated atoms escape the solvent cage to recombine with an atom other than their original partner. But a more striking feature of their results is the signature of structural variation in the solvent during the reaction (Fig. 1). When the reaction is probed on a spatial scale of around $0.6 \mathrm{~nm}$, the solvent structure is seen to change slowly for about $20 \mathrm{~ns}$, and then to change rapidly over a period of about $50 \mathrm{~ns}$. But when the reaction is probed on a smaller spatial scale of $0.15 \mathrm{~nm}$, the solvent structure changes at about the same rate for several hundred nanoseconds. The surprising implication is that although the initial change in solvent structure, arising from the deposition of energy from the recombining iodine atoms, occurs in less than $200 \mathrm{ps}$, the transformation of that initial change to bulk thermal expansion does not occur for another 10,000 ps.

It is interesting to speculate that the solvent-structure change is a monitor of a coherent excitation that later decays, but this remains to be determined. If, indeed, the motion of an excited molecule and its surrounding shell of solvent molecules is coherent for a reasonable fraction of the reaction time, then our picture of how solute and solvent interact during a reaction will need to be modified. In this case, the solvent is much more than just a source of random energy exchanges with the reactant. Rather, solute and solvent act as a supermolecule, defining the reaction rate and, possibly, also the reaction mechanism.

Stuart A. Rice is in the Department of Chemistry and the James Franck Institute, University of

Chicago, 5640 S Ellis Avenue, Chicago,

Illinois 60637, USA.

e-mail: sarice@uchicago.edu

Solar System

\title{
Captain Cook and the black drop
}

At intervals, Venus passes directly between the Earth and the Sun, making a transit across the Sun's bright disk. On 8 June, an entire transit of Venus will be visible from most of Asia, Africa and Europe. Australia will experience the transit at around sunset, and across the eastern side of the Americas it will occur around sunrise.

Johannes Kepler, as he plotted the motions of the heavens, was the first to realize that such transits would occur. However, his predicted transit of 1631 was not visible in Europe. The first recorded sighting of a transit was made, in north-west England, by Jeremiah Horrocks, whose own calculations had pinpointed a repeat of the spectacle on 4 December 1639. The intervals between transits follow an unusual pattern: 8 years, then 121.5 , then 8 , then 105.5 years, and will do so until 2984.

Seventeenth-century astronomers hoped that observations of a transit of Venus would allow them to obtain an accurate measure of the distance from the Earth to the Sun. The concept was eventually formally published in 1716 by Edmund Halley, who predicted from where the transit of 1761 should be viewed so that measurements of the transit times could be used to calculate the Sun's distance. But his predictions were somewhat awry: Halley mistakenly used a plus sign in his calculation where there should have been a minus.

Mathematical fallibility aside, in fact the transit of Venus has never yielded an accurate measure of the Earth-Sun distance. The calculation is hampered by what is known as the 'black drop problem'. The exact moment at which the dark disk of Venus is fully on the disk of the Sun is hard to ascertain, because the planet's disk seems to stay connected to the dark edge of the Sun: the disk appears as a black drop rather than a perfect circle. Only last year was this effect attributed to the way in which the Sun's brightness lessens at its visible edge, in combination with the natural blurring caused by a telescope.

The difficulty of timing a transit was also noted by Captain James Cook (pictured), on his first roundthe-world voyage on the Endeavour. In 1769 , before reaching the east coast of Australia, Cook was at Tahiti. On Saturday 3 June, he wrote

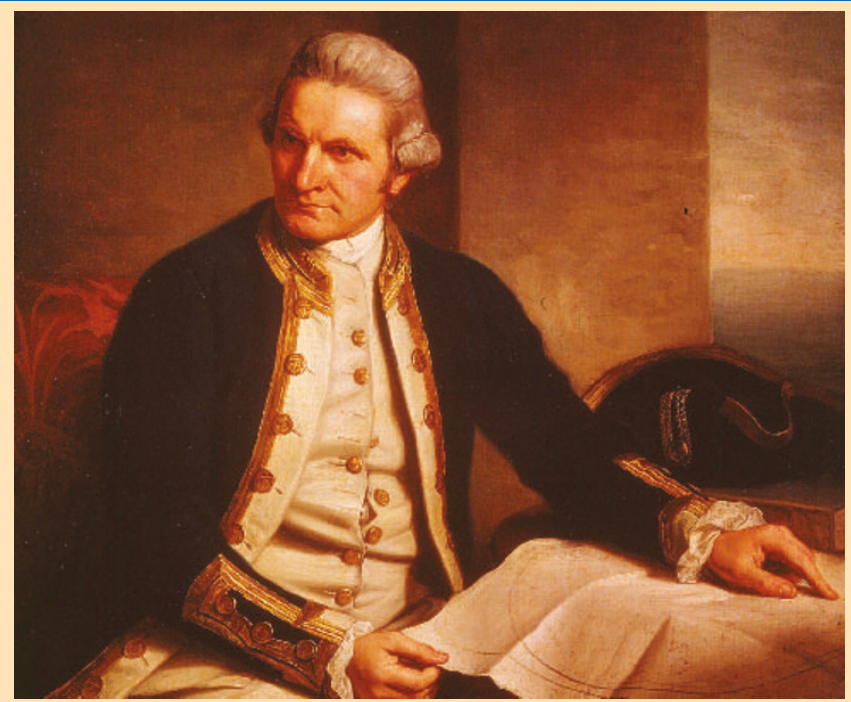

in his logbook: "This day proved as favourable to our purpose as we could wish. Not a Cloud was to be seen the whole day, and the Air was perfectly Clear, so that we had every advantage we could desire in observing the whole of the Passage of the planet Venus over the Sun's Disk. We very distinctly saw an Atmosphere or Dusky shade round the body of the planet, which very much disturbed the times of the Contact, particularly the two internal ones. Dr. Solander observed as well as Mr. Green and myself, and we differ'd from one another in Observing the times of the Contact much more than could be expected."

By 1882, transit fever had captured both the public imagination and the newspaper headlines. The San Francisco Chronicle reported that "Many of the residents of San Francisco were noticed yesterday with a piece of smoked glass to their eye, looking curiously at the sun". William Harkness, then director of the US Naval Observatory in Washington DC, had warned on the eve of that transit that "there will be no other till the twenty-first century of our era has dawned upon the Earth, and the June flowers are blooming in 2004".

The transit begins shortly after 5:00 Universal Time on 8 June. To view it, the simplest of pinhole cameras will suffice - weather permitting, of course. Alison Wright 